\title{
Evaluación mediante software de la efectividad de los anclajes de cinturón de seguridad de un asiento comercial de autobús según la regulación ECE R14
}

\section{(Software evaluation of the effectiveness of the seatbelt anchorages of a commercial bus seat according to regulation ECE R14)}

\author{
Arroba Arroba César Hernán¹, Núñez Núñez Diego Fernando
}

\begin{abstract}
Resumen:
El cinturón de seguridad es un elemento importante para mantener al ocupante sujeto al asiento durante un accidente; sin embargo, si el anclaje del cinturón no resiste durante la colisión, el ocupante es expulsado del asiento, por tanto, es indispensable analizar su efectividad. El objetivo de este trabajo es evaluar la resistencia de los anclajes para cinturones de seguridad de dos y tres puntos de anclaje de un asiento comercial de autobuses, aplicando la Regulación de la Comisión Económica de las Naciones Unidas para Europa (ECE-R14). Las propiedades de los materiales de la estructura del asiento y de los anclajes del cinturón se obtuvieron mediante ensayos de tracción y flexión. Estos valores se utilizaron para elaborar un modelo de elementos finitos utilizando el material Piecewise Linear Isotropic Plasticity de Ansys - LsDyna. Los anclajes se evaluaron aplicando fuerzas en los bloques pélvico y torácico sujetos a través del cinturón según lo especificado en la regulación. En el análisis los anclajes presentaron grandes deformaciones llegando a la ruptura, modificando la forma y posición de los anclajes e incrementando refuerzos estructurales en áreas de mayor solicitación los anclajes cumplieron con la regulación. Este trabajo pretende crear una metodología para la industria de asientos que permita evaluar sus modelos previos a homologaciones.
\end{abstract}

Palabras clave: asiento de autobús; anclajes de cinturón de seguridad; resistencia; seguridad pasiva; análisis por elementos finitos; ECE R14.

\begin{abstract}
:
The seatbelt is an important element to keep the occupant subject to the seat during an accident. However, if the seatbelt anchorage does not resist the occupant during a crash, it is ejected from the seat. Therefore, it is essential to analyze its effectiveness. The purpose of this work is to assess the strength of the anchorages for safety belts of 2 and 3 anchorage points of a commercial bus seat, applying the Regulation of the United Nations Economic Commission for Europe (ECE-R14). The materials properties of the seat structure and the belt anchorages were obtained by tensile and bending tests. A finite element model was created using Piecewise Linear Isotropic Plasticity from Ansys - LsDyna. The anchors were evaluated by applying forces on the pelvic and thoracic blocks fastened through the belt as specified in the regulation. In the analysis, the anchors presented large deformations reaching the rupture. Modifying the shape and position of the anchors and increasing structural reinforcements in high-stress areas, the anchors complied with the regulation. This work aims to create a methodology for the seating industry that allows assessing their models prior to homologations.
\end{abstract}

Keywords: seat; seatbelt anchorage; strength; safety; finite element; ECE R14.

\footnotetext{
1 Universidad Técnica de Ambato - Carrera de Ingeniería Mecánica, Ambato, Ecuador ( \{ch.arroba, df.nunez\} @uta.edu.ec.
} 


\section{Introducción}

El autobús de pasajeros es considerado uno de los medios de transporte más importantes hoy en día (Siwadamrongpong, Rooppakhun, Burakorn, \& Murachai, 2013)(Patil \& Kumar, 2016). Solo en Estados Unidos en 2010 los ciudadanos en promedio viajaron 21000 kilómetros al año de los cuales cerca del 8\% lo hicieron mediante autobuses de servicio público (Yuce, Karpat, Yavuz, \& Sendeniz, 2014). En el Ecuador, aunque algo menor, las cifras siguen siendo importantes; en el año 2016 aproximadamente unos 690000 vehículos transportaron personas donde alrededor del 3\% correspondieron a autobuses de servicio público (INEC, 2016), esto supone una serie de problemas medioambientales y de riesgos de muerte o lesiones en las carreteras por la gran cantidad de personas que viajan en este tipo de medio de transporte (Siwadamrongpong et al., 2013). Según algunos reportes, en el mundo 1.2 millones de personas mueren al año por accidentes de tránsito y 50 millones resultan con heridas moderadas o graves (Ogundele, Ifesanya, Adeyanju, \& Ogunlade, 2013). De acuerdo a las estadísticas del Banco Mundial, el Ecuador en el 2015 tuvo una tasa de mortalidad causada por accidentes de tránsito del 20.7 por cada 100000 personas, la cual es mayor que la media de la región (Bank, 2015). Estos datos se corroboran con los reportados por la Agencia Nacional de Tránsito - ANT donde desde el año 2015 al 2017 existió casi un total de 6300 fallecidos y 22000 heridos por accidentes de tránsito de los 94000 siniestros presentados (ANT, 2016); considerando que dentro de las lesiones están las denominadas catastróficas como daños cerebrales, paraplejias y cuadriplejias (Veeranjaneyulu \& Ravikanth, 2015); es decir, en Ecuador 6 personas mueren cada día en accidentes de tránsito.

Para solucionar esta problemática se deben tomar en cuenta el confort y la seguridad de los pasajeros dentro de un autobús. En este campo el asiento es quizá el elemento más importante para la seguridad y confort del pasajero durante el viaje (Siwadamrongpong et al., 2013)(Patil \& Kumar, 2016). Estos asientos tienen varios componentes que ayudan en la seguridad pasiva de los pasajeros debido a que juegan un papel preponderante para disminuir la tasa de mortalidad de accidentes de tránsito. Un estudio de la National Highway Traffic Safety Administration (NHTSA) indica que el uso de cinturón de seguridad disminuye la tasa de mortalidad en un accidente de tránsito en un $45 \%$ y en un $50 \%$ la probabilidad de lesiones graves (NHTSA, 2015). Dentro de los componentes de seguridad pasiva en los autobuses el cinturón de seguridad se considera un dispositivo de alta efectividad al momento de proteger al pasajero en una colisión debido a que no solo salva vidas, sino que disminuye el riesgo de lesiones de gravedad. Los cinturones de seguridad se sujetan o anclan firmemente al vehículo de tal manera que cumplan su función; por lo tanto, es imprescindible que los anclajes garanticen el buen funcionamiento del cinturón de seguridad para evitar lesiones o la muerte de los pasajeros en caso de un accidente ( $\mathrm{Li}$, Peng, Rong, Men, \& Zhao, 2013).

\subsection{Reglamentación para evaluación de asientos y de anclajes de cinturones de seguridad}

A nivel mundial existen diferentes regulaciones para evaluar la efectividad de los asientos de pasajeros dentro de ellas las más importantes son las regulaciones de la Comisión Económica de las Naciones Unidas para Europa (ECE por sus siglas en inglés) en las cuales se definen una serie de normas, como es el caso del Reglamento R66 destinado a evaluar la resistencia al vuelco (roll over) de los autobuses, el Reglamento R80 para la resistencia de la estructura y anclajes de los asientos de pasajeros y el reglamento (UNECE, 2015) que se encarga de evaluar la conformidad de la resistencia mecánica de los anclajes de los cinturones de seguridad, por ser esta última una reglamentación muy exigente en ella se indica que un asiento que apruebe a conformidad sus requisitos básicos es apto para resistir las condiciones del ensayo del reglamento $R 80$ (INEN 2708 su equivalente en la normativa ecuatoriana). En cuanto a los anclajes de los 
cinturones de seguridad, las regulaciones más utilizadas para este propósito son la ECE R14 (europea) y la FMVSS210 (norteamericana) las cuales proponen ensayos físicos para asegurar la resistencia de los puntos de anclaje. Existen dos diferencias principales entre estas reglamentaciones, la primera, se refiere a la aplicación de la carga, mientras que en la ECE R14 se aplican las cargas lo más rápido que se pueda con un sostenimiento de 0.2 segundos, en la FMVSS210 la aplicación de la carga se la hace de 1 a 30 segundos con un sostenimiento de 10 segundos, de tal manera que en esta última se puede considerar un análisis quasi-estático a diferencia de la primera donde los efectos dinámicos son evidentes. La segunda diferencia es respecto a la aplicabilidad de la regulación, en la ECE R14 se clasifica a los vehículos a motor por su peso y se requieren diferentes cargas para el ensayo, mientras que en la FMVSS210, la misma carga es aplicada a cualquier tipo de vehículo (UNECE, 2015). La normativa ecuatoriana equivalente a estas regulaciones es la NTE INEN 2704. En este trabajo se utilizó la metodología descrita en la regulación ECE R14 por ser la regulación más importante a nivel mundial en este campo y que además permite evaluar los cinturones con dos o tres puntos de anclaje. (Yuce et al., 2014). En general en los autobuses se usan estos dos tipos de configuraciones de anclajes diferenciando al conductor y pasajeros de acuerdo con la fila en que se ubican.

\subsection{Requisitos mínimos de cumplimiento de la Regulación ECE R14}

Según la regulación ECE R14, la finalidad de los ensayos de resistencia de los anclajes de los cinturones de seguridad es determinar: (i) que el ocupante u ocupantes de los asientos queden correctamente retenidos por el uso del cinturón de seguridad, (ii) que el asiento y los anclajes de los cinturones de seguridad sean suficientemente resistentes y (iii) que el ocupante o los ocupantes del asiento no sufran lesiones de gravedad. Adicionalmente los anclajes deberán estar proyectados, construidos y colocados de manera que permitan la instalación de un cinturón de seguridad adecuado de dos o tres puntos, reduzcan al mínimo el riesgo de deslizamiento del cinturón cuando esté correctamente colocado y minimicen el riesgo de deterioro de la correa por contacto con las partes rígidas salientes (UNECE, 2015).

El propósito de este estudio fue evaluar por medio de análisis dinámico explícito por elementos finitos la resistencia de los anclajes del cinturón de seguridad de un asiento comercial de autobús acorde con los requerimientos de la regulación ECE R14 usando el "método estático", que recomienda la regulación, que representa la colisión frontal del autobús. En este sentido resulta un hecho relevante evaluar a los asientos comerciales de la industria ecuatoriana debido a que existen reportes de inobservancias en cuando al cumplimiento de dimensiones mínimas (Reyes, Rosales, Guzmán, \& Báez, 2017). Adicionalmente a esto, se propone un rediseño en la estructura del asiento para el cumplimiento de dicha regulación, de tal manera que constituya una metodología para la industria de los asientos para que evalúe sus modelos y realice las modificaciones necesarias para el cumplimiento de las regulaciones vigentes.

\section{Metodología}

\subsection{Asiento y materiales}

Los experimentos se realizaron en un asiento comercial de autobús de $1000 \mathrm{~mm}$ de ancho de un fabricante de la zona centro de Ecuador que incluyen anclajes para cinturones de seguridad de dos y tres puntos, ver Figura 1 (a). De este se eliminaron las espumas y tejidos que recubren la estructura enfocando el análisis en los anclajes del cinturón con la estructura del asiento. Los materiales utilizados en la fabricación del asiento se muestran en la Tabla 1. 
Tabla 1. Materiales de la estructura del asiento

\begin{tabular}{|c|c|c|c|}
\hline Parte & Material & Descripción & Dimensiones [mm] \\
\hline $\begin{array}{l}\text { Anclaje del cinturón de } \\
\text { tres puntos }\end{array}$ & ASTM A 36 & Tubo estructural redondo & $\mathrm{D}=22, \mathrm{e}=2$ \\
\hline Espaldar & $\begin{array}{l}\text { ASTM A } 500 \\
\text { GRADO A }\end{array}$ & Tubo estructural redondo & $\mathrm{D}=25.4, \mathrm{e}=2$ \\
\hline Cinturón de seguridad & Poliéster & Correa & $40 \times 1.3$ \\
\hline Articulación & ASTM A 36 & Pernos grado ISO 8.8 & M 12 \\
\hline Cojín & ASTM A 36 & $\begin{array}{l}\text { Tubo estructural redondo } \\
\text { T estructural } \\
\text { Ángulo }\end{array}$ & $\begin{array}{l}\mathrm{D}=25.4, \mathrm{e}=2 \\
20 \times 20 \times 3 \\
25 \times 25 \times 4\end{array}$ \\
\hline $\begin{array}{l}\text { Anclaje del cinturón de } \\
\text { dos puntos }\end{array}$ & $\begin{array}{l}\text { ASTM A } 500 \\
\text { GRADO A }\end{array}$ & Tubo estructural redondo & $\mathrm{D}=22, \mathrm{e}=2$ \\
\hline Base & $\begin{array}{l}\text { ASTM A } 500 \\
\text { GRADO A }\end{array}$ & $\begin{array}{l}\text { Tubo redondo } \\
\text { Tubo cuadrado }\end{array}$ & $\begin{array}{l}D=25.4, e=2 \\
25 \times 25 \times 2\end{array}$ \\
\hline Pata & ASTM A 36 & $\begin{array}{l}\text { Ángulo } \\
\text { Tubo ovalado }\end{array}$ & $\begin{array}{l}30 \times 30 \times 4 \\
50 \times 40 \times 2\end{array}$ \\
\hline Anclaje en la carrocería & ASTM A 36 & Ángulo & $30 \times 30 \times 4$ \\
\hline
\end{tabular}

Del acero ASTM A500 se elaboraron cinco probetas planas de $510 \mathrm{~mm} \times 250 \mathrm{~mm} \times$ $2 \mathrm{~mm}$, evaluándose la resistencia a la tracción según la norma ISO 6892 "Materiales metálicos. Ensayo de tracción. Parte 1: Método de ensayo a temperatura ambiente" (ISO6892, 2016). Los ensayos se realizaron en una máquina universal Metrotec Serie MTE50 de los que se obtuvieron valores de esfuerzo desplazamiento; estos experimentos se replicaron mediante simulación utilizando los resultados del ensayo de tracción y asignando al modelo de material Piecewise Linear Isotropic Plasticity de Ansys Ls-Dyna, con este procedimiento se validó el modelo de material utilizado en los análisis de los anclajes.

\subsection{Procedimiento de análisis de la resistencia de los anclajes}

La resistencia de los anclajes para cinturones de dos y tres puntos se evaluaron según ECE-R14 numeral 6 (UNECE, 2015). Las cargas aplicadas en los dispositivos de tracción pélvicos y torácicos, sujetos al asiento a través del cinturón de seguridad se indican en la Tabla 2. La configuración de los dispositivos para la aplicación de las cargas se muestra en la Figura 1 (b).

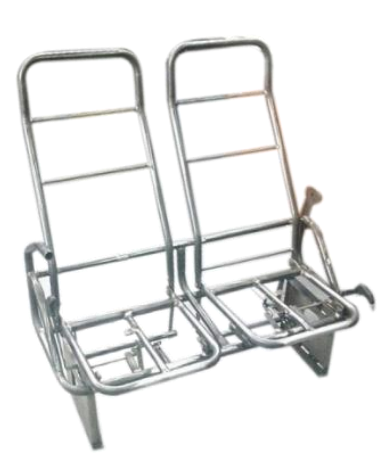

(a)

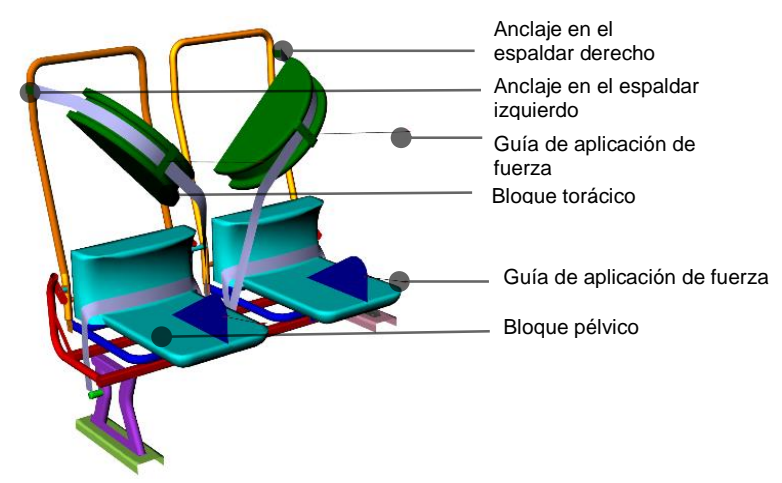

(b)

Figura 1. Asiento comercial de autobús. (a)Estructura de asiento que actualmente se fabrica (b) Disposición de los bloques pélvico y torácico en el asiento. 
Tabla 2. Carga aplicada en el bloque pélvico y torácico

\begin{tabular}{cccccccc}
\hline $\begin{array}{c}\text { Tipo de } \\
\text { cinturón }\end{array}$ & $\begin{array}{c}\text { Bloque } \\
\text { pélvico } \\
{[\mathrm{N}]}\end{array}$ & $\begin{array}{c}\text { Bloque } \\
\text { torácico } \\
{[\mathrm{N}]}\end{array}$ & $\begin{array}{c}\text { Inercia por } \\
\text { la masa del } \\
\text { asiento }[\mathrm{N}]\end{array}$ & $\begin{array}{c}\text { Total } \\
\text { bloque } \\
\text { pélvico } \\
{[\mathrm{N}]}\end{array}$ & $\begin{array}{c}\text { Total } \\
\text { bloque } \\
\text { torácico } \\
{[\mathrm{N}]}\end{array}$ & $\begin{array}{c}\text { Ángulo de } \\
\text { aplicación }\left[{ }^{\circ}\right]\end{array}$ & $\begin{array}{c}\text { Tiempo de } \\
\text { aplicación } \\
{[\mathrm{s}]}\end{array}$ \\
\hline 3 puntos & 4500 & 4500 & 1908 & 6408 & 4500 & 10 & 0.2 \\
\hline 2 puntos & 7400 & 0 & 1908 & 9308 & 0 & 10 & 0.2 \\
\hline
\end{tabular}

La geometría de la estructura del asiento, cinturón de seguridad, bloque pélvico y torácico se modelaron mediante CAD; las dimensiones y características se tomaron del asiento comercial y los bloques pélvico y torácico según las especificaciones del reglamento. Las simulaciones se desarrollaron en los software ANSYS Research, LS DYNA. Para el mallado de los componentes se utilizaron elementos tipo Shell de forma triangular y rectangular, la dimensión máxima del tamaño de elemento por lado fue $5 \mathrm{~mm}$ en la estructura y $25 \mathrm{~mm}$ en el bloque pélvico y torácico, la formulación de los elementos se utilizó el tipo 2 (ELFORM =2), el factor de corrección cortante 0.83333 (SHRF=0.83333), los números de puntos de integración de 3 (NPI=3), el espesor de los elementos se considera uniforme y correspondiente a cada parte del asiento (Shi, Wang, \& Xiao, 2017).

Tabla 3. Modelos de materiales utilizados en Ansys-LsDyna y sus especificaciones

\begin{tabular}{|c|c|c|c|c|c|c|c|}
\hline \multicolumn{8}{|c|}{ Propiedades de MAT_ELASTIC para el cinturón de seguridad } \\
\hline $\begin{array}{l}\text { Densidad } \\
{\left[\mathrm{kg} / \mathrm{m}^{3}\right]}\end{array}$ & $\begin{array}{l}\text { Módulo de } \\
\text { Young } \\
\text { [MPa] }\end{array}$ & $\begin{array}{l}\text { Relación } \\
\text { de Poisson }\end{array}$ & & & & & \\
\hline 1390 & 110000 & 0.3 & & & & & \\
\hline \multicolumn{8}{|c|}{ Propiedades de MAT_ELASTIC para las articulaciones } \\
\hline $\begin{array}{c}\text { Densidad } \\
{\left[\mathrm{kg} / \mathrm{m}^{3}\right]}\end{array}$ & $\begin{array}{l}\text { Módulo de } \\
\text { Young } \\
\text { [MPa] }\end{array}$ & $\begin{array}{l}\text { Relación } \\
\text { de Poisson }\end{array}$ & & & & & \\
\hline 7890 & 200000 & 0.3 & & & & & \\
\hline \multicolumn{8}{|c|}{ Propiedades del MAT_PIECEWISE_LINEAR_PLASTICITY para el acero ASTM A500 } \\
\hline $\begin{array}{l}\text { Densidad } \\
{\left[\mathrm{kg} / \mathrm{m}^{3}\right]}\end{array}$ & $\begin{array}{l}\text { Módulo de } \\
\text { Young } \\
\text { [MPa] }\end{array}$ & $\begin{array}{l}\text { Relación } \\
\text { de Poisson }\end{array}$ & $\begin{array}{l}\text { Deformación } \\
\text { plástica de } \\
\text { rotura }\end{array}$ & $\begin{array}{c}\text { Esfuerzo } \\
\text { de } \\
\text { fluencia } \\
\text { [MPa] }\end{array}$ & $\begin{array}{l}\text { Deformación } \\
\text { de Inicio de } \\
\text { falla }\end{array}$ & $\begin{array}{c}\text { C velocidad } \\
\text { de } \\
\text { deformación }\end{array}$ & $\begin{array}{c}\text { P velocidad } \\
\text { de } \\
\text { deformación }\end{array}$ \\
\hline 7890.00 & 200000.00 & 0.30 & 0.283 & 320.00 & 0.370 & 40.00 & 5.00 \\
\hline \multicolumn{8}{|c|}{ Propiedades del MAT_PIECEWISE_LINEAR_PLASTICITY para el acero ASTM A500 comportamiento no lineal } \\
\hline 0.0000 & 0.01092 & 0.03959 & 0.04388 & 0.04817 & 0.1431 & 0.2545 & 0.4000 \\
\hline 320.0 & 320.00 & 347.16 & 351.22 & 355.28 & 382.07 & 341.91 & 0.0 \\
\hline \multicolumn{8}{|c|}{ Propiedades del MAT_PIECEWISE_LINEAR_PLASTICITY para el acero ASTM A36 } \\
\hline $\begin{array}{l}\text { Densidad } \\
{\left[\mathrm{kg} / \mathrm{m}^{3}\right]}\end{array}$ & $\begin{array}{l}\text { Módulo de } \\
\text { Young } \\
\text { [MPa] }\end{array}$ & $\begin{array}{l}\text { Relación } \\
\text { de Poisson }\end{array}$ & $\begin{array}{l}\text { Deformación } \\
\text { plástica de } \\
\text { rotura }\end{array}$ & $\begin{array}{c}\text { Esfuerzo } \\
\text { de } \\
\text { fluencia } \\
\text { [MPa] }\end{array}$ & $\begin{array}{l}\text { Deformación } \\
\text { de Inicio de } \\
\text { falla }\end{array}$ & $\begin{array}{c}\text { C velocidad } \\
\text { de } \\
\text { deformación }\end{array}$ & $\begin{array}{c}\text { P velocidad } \\
\text { de } \\
\text { deformación }\end{array}$ \\
\hline 7890.00 & 200000.00 & 0.30 & 0.283 & 356.00 & 0.283 & 40.00 & 5.00 \\
\hline \multicolumn{8}{|c|}{ Propiedades del MAT_PIECEWISE_LINEAR_PLASTICITY para el acero ASTM A36 comportamiento no lineal } \\
\hline 0.0 & 0.0205 & $0.033 \overline{6}$ & $\overline{0.0605}$ & 0.0793 & 0.296 & 0.374 & 0.3844 \\
\hline 356 & 356 & 383.77 & 393.80 & 400.49 & 511.98 & 429.50 & 0.00 \\
\hline
\end{tabular}

Las condiciones de frontera se aplicaron en circunstancias de funcionamiento del asiento en el autobús. Los extremos inferiores de los perfiles estructurales del piso ubicados bajo la pata del asiento al lado izquierdo y el anclaje al zócalo del lado derecho se restringieron todos sus grados de libertad. A todos los elementos del asiento se aplicó contacto automático entre superficies ( ${ }^{*}$ CONTACT AUTOMATIC SINGLE SURFACE ID) y además se restringió la penetración entre partes. Los bloques pélvicos y torácicos se consideraron con propiedades de cuerpos totalmente rígidos (Huelke \& Compton, 1995). 
Las propiedades de los materiales utilizados en los componentes de los asientos se definieron en los modelos de materiales MAT ELASTIC y MAT PIECEWISE LINEAR PLASTICITY , este último permite definir el comportamiento en la zona elástica y plástica (Heo, Kim, Song, Yang, \& Im, 2011). Estas propiedades se muestran en la Tabla 3.

En el caso que la estructura del asiento y sus anclajes no cumplan los requerimientos de la regulación ECE R14 se propondrá una alternativa para que la estructura del asiento esté en conformidad por lo estipulado en los ensayos

\section{Resultados y Discusión}

La curva promediada de los ensayos de tracción de las probetas de acero ASTM A500, comparada con la curva obtenida de simulación, presentaron buena aproximación según se muestra en la Figura 2, el error promediado calculado es $6.5 \%$ y el error máximo es $12.55 \%$.

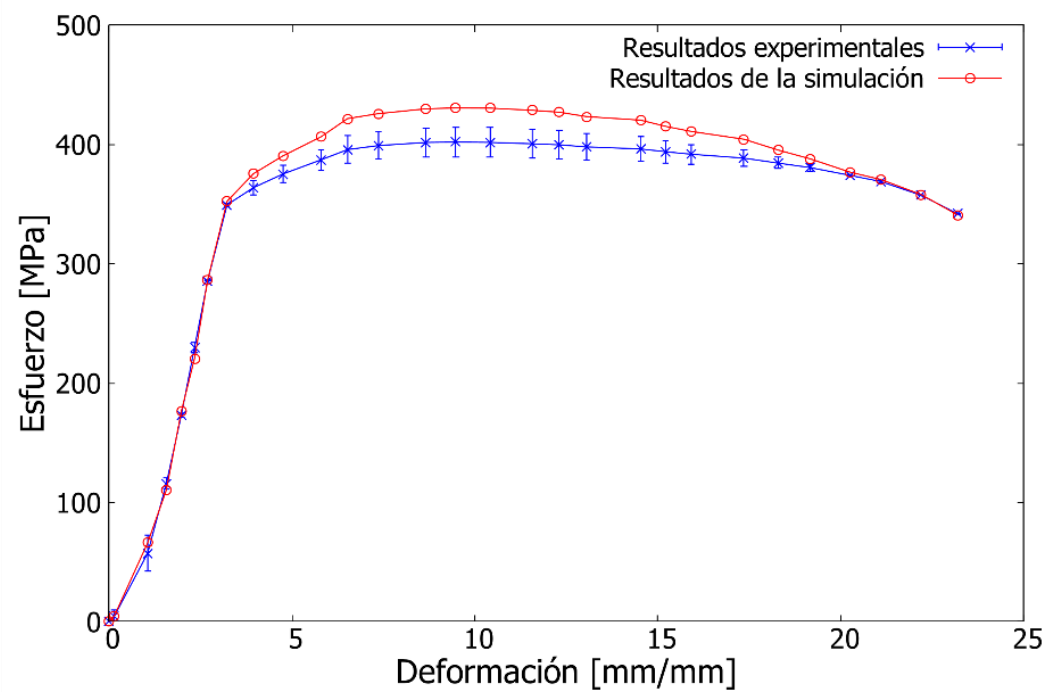

Figura 2. Resultados experimentales y simulados del ensayo a tracción del acero A500

\subsection{Análisis de la resistencia de los anclajes}

De acuerdo con las condiciones establecidas para evaluar la resistencia de los anclajes del cinturón de seguridad de dos y tres puntos, sin modificaciones del diseño original, las estructuras de los asientos se desplazaron de su posición original a la mostrada en la Figura 3, este desplazamiento resulta de la deformación de sus componentes por acción de la carga aplicada al bloque pélvico y torácico según el tipo de cinturón. El anclaje central a la base para los dos tipos de cinturón tuvo mayor desplazamiento.

La Figura 4 (a) presenta las curvas de la distribución de los esfuerzos máximos equivalentes en función del tiempo de simulación de los anclajes para el cinturón de dos puntos, en esta se obtiene que el valor máximo de $830 \mathrm{MPa}$ alcanza el anclaje central sobrepasando el límite de rotura del material, esto se corrobora en la Figura 4(b); en la curva de deformación plástica efectiva en el tiempo de 0.03 segundos, la deformación iguala al valor de rotura del material y a los 0.08 segundos sobrepasa este valor llegando a la falla del anclaje. La deformación plástica efectiva en el anclaje que está conectado a la pata llega a 0.28 y el anclaje lateral sujeto a la base llega a 0.091 ; estos valores son inferiores a la ruptura; sin embargo, estos anclajes presentan deformación permanente. 


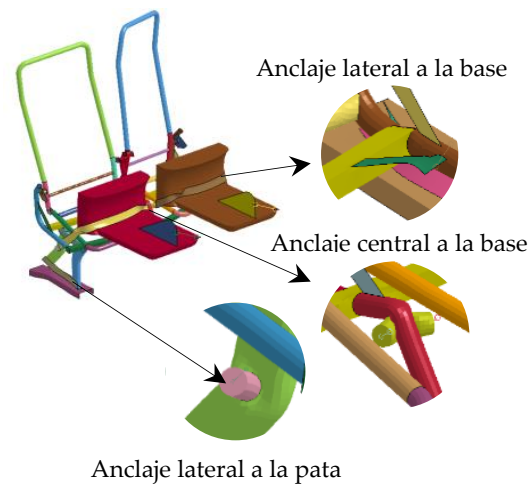

a) Cinturón de dos puntos

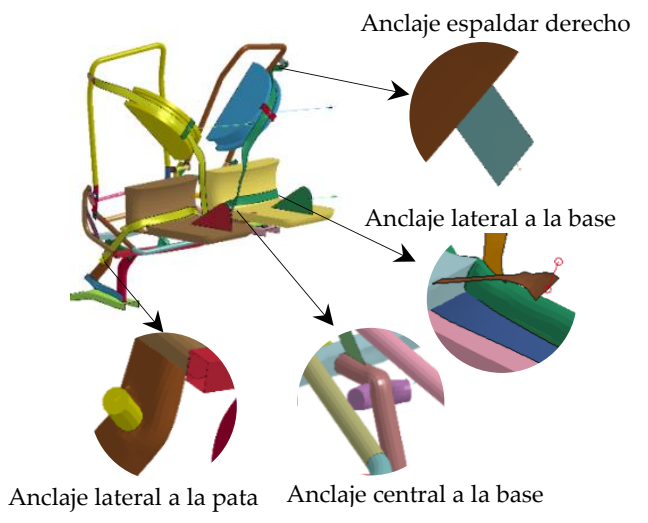

b) Cinturón de tres puntos

Figura 3. Desplazamiento de la estructura del asiento $t=0.2 \mathrm{~s}$

En el caso de los anclajes para cinturones de tres puntos el anclaje central a la base alcanza el mayor valor de esfuerzo máximo equivalente de $700 \mathrm{MPa}$, Figura 4(c) y la deformación plástica efectiva de esta parte es 0.13 que no alcanza valores de ruptura, Figura $4(d)$. El anclaje lateral a la base y el anclaje lateral a la pata presenta valores menores de esfuerzo y deformación plástica efectiva manteniéndose sujeto a la estructura del asiento. En los anclajes que están sujetos a los espaldares del asiento no presentaron deformación plástica.

Los anclajes para el cinturón de dos puntos luego de realizado el ensayo y comparando con los requerimientos de cumplimiento descrito en el ECE-R14 numeral 7; el anclaje que se encuentra en la parte central del asiento no cumple por presentar ruptura. Los anclajes para el cinturón de tres puntos no presentaron ruptura; no obstante, la estructura del asiento presenta cambios considerables de su forma especialmente en la base y pata de la estructura. El espaldar izquierdo y derecho presentaron un desplazamiento superior a los $10^{\circ}$ que permite la regulación ECE-R14.

\subsection{Modificación de diseño original}

Se sabe durante años que el diseño de asientos debe guardar un compromiso entre rigidez y absorción de energía en el impacto (Olschinka, Schumacher, \& Riedel, 2006). Varias investigaciones (Yuce et al., 2014)(Li et al., 2013)(Jung, Cho, Lee, Oh, \& Kwon, 2012) han tratado las modificaciones desde varias perspectivas, algunas enfocadas al cambio de material (aleaciones ligeras o compuestos), de espesores, nuevos elementos o incluso puntos de soldadura para rigidizar la estructura de tal manera que no pierdan la capacidad de superar los requerimientos de las regulaciones vigentes.

En este trabajo se reemplazaron ciertos elementos críticos del asiento para rigidizar la estructura; el cambio de material resulta una opción a posteriori debido a la poca oferta del mercado local en aceros de alta resistencia. No se consideró el incremento de espesores de los perfiles ya que significa al menos un 30\% más de peso que en un autobús de 50 pasajeros resulta en un incremento de mínimo $300 \mathrm{~kg}$ al peso total del autobús, aproximadamente un $2 \%$ adicional; lo que implica según [3] un incremento de gasto de combustible de 0.6 litros por cada $100 \mathrm{~km}$ recorridos.

Para mejorar la resistencia de los anclajes se modificó el diseño original sin que afecte la secuencia de fabricación en la línea de producción. Se eliminaron los anclajes originales y se remplazó por placas triangulares de $4 \mathrm{~mm}$ de espesor en las esquinas de la estructura base donde se realizaron orificios para alojar los pernos de anclaje de los cinturones (ver Figura 5); además, en la estructura base de asiento se incrementaron dos tubos adyacentes al anclaje central con el fin de rigidizarla. Para el cinturón de tres puntos 
en los anclajes que están conectados al espaldar no se realizaron modificaciones (ver Figura 5).

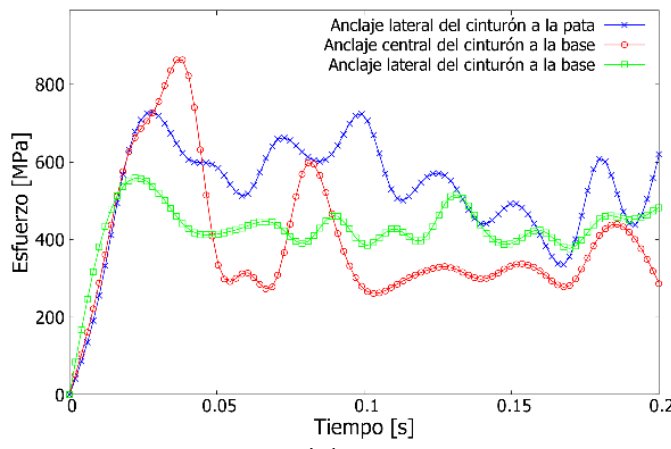

(a)

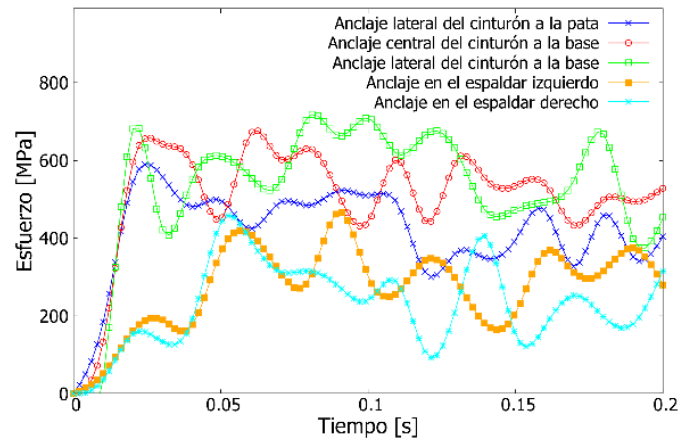

(d)

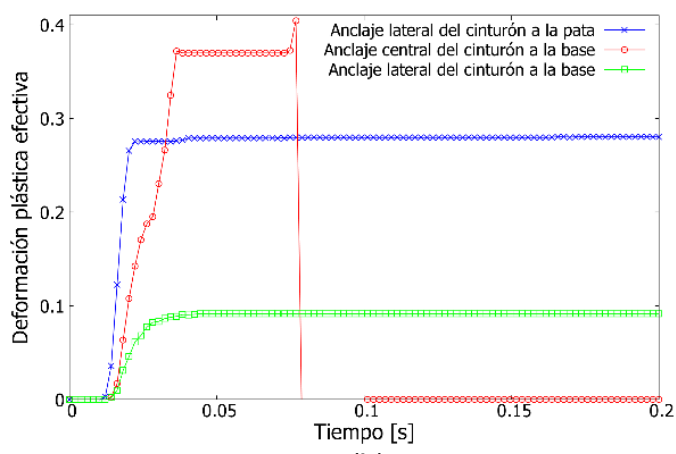

(b)

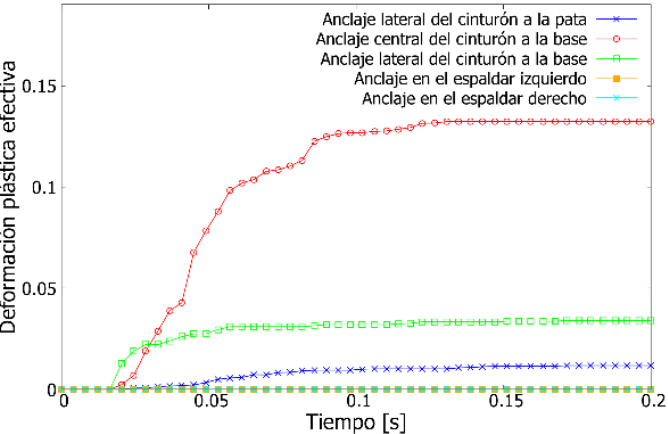

(e)

Figura 4. Resistencia de los anclajes de cinturón. (a) Esfuerzos en los anclajes del cinturón de 2 puntos (b) Deformación plástica efectiva en los anclajes del cinturón de 2 puntos (c) Esfuerzos en los anclajes del cinturón de 3 puntos (d) Deformación plástica efectiva en los anclajes del cinturón de 3 puntos.

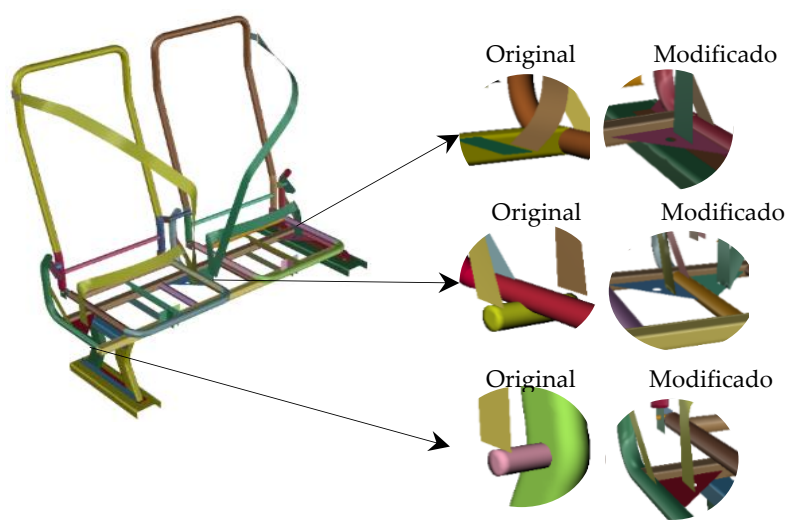

Figura 5. Modificaciones en los anclajes

\subsection{Resistencia de los anclajes modificados}

De acuerdo con las condiciones establecidas para evaluar la resistencia de los anclajes del cinturón de seguridad de dos puntos y con las modificaciones de la estructura del asiento y anclajes según la Figura 5, se disminuyó en un $30 \%$ el desplazamiento de las partes de la estructura del asiento, Figura 6 (izq.). En los anclajes del cinturón de seguridad de tres puntos la estructura base y espaldares se desplazaron $25 \%$ menos en relación con el diseño original; todos los anclajes resistieron el ensayo sin presentar gran cambio en su forma, Figura 6 (der.)

La Figura 7(a) presenta curvas de la distribución de los esfuerzos equivalentes en función del tiempo para los anclajes del cinturón de dos puntos, el esfuerzo en todos los 
anclajes no supera el límite de fluencia del material de $320 \mathrm{MPa}$. De estos anclajes, el anclaje lateral derecho alcanza un esfuerzo máximo y mínimo de $290 \mathrm{MPa}$ y $120 \mathrm{MPa}$ respectivamente. Esto se debe a que la sujeción del asiento a la estructura de la carrocería del autobús es más rígida restringiendo el desplazamiento en la zona del anclaje del cinturón. Para el cinturón de tres puntos el anclaje lateral derecho e izquierdo mostraron deformación permanente con valores muy pequeños de deformación plástica efectiva, alrededor de 0.009 y 0.001 respectivamente, ver Figura 7(c). Los demás anclajes no superaron el límite de fluencia.
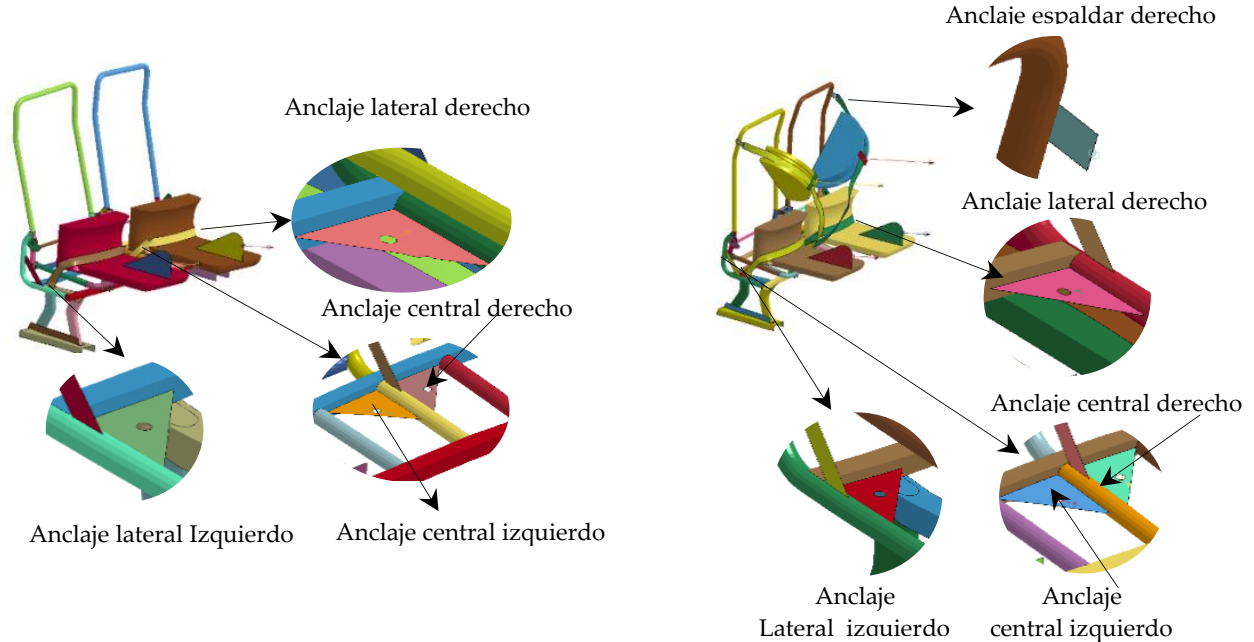

Figura 6. Deslazamiento de los anclajes para $t=0.2 \mathrm{~s}$

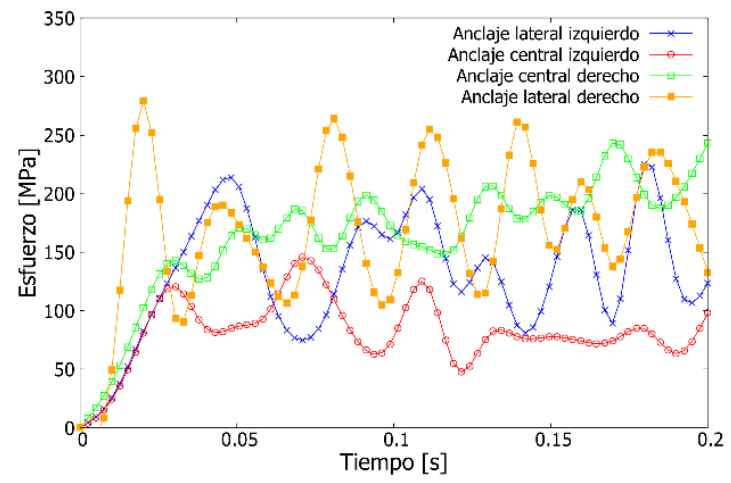

(a)

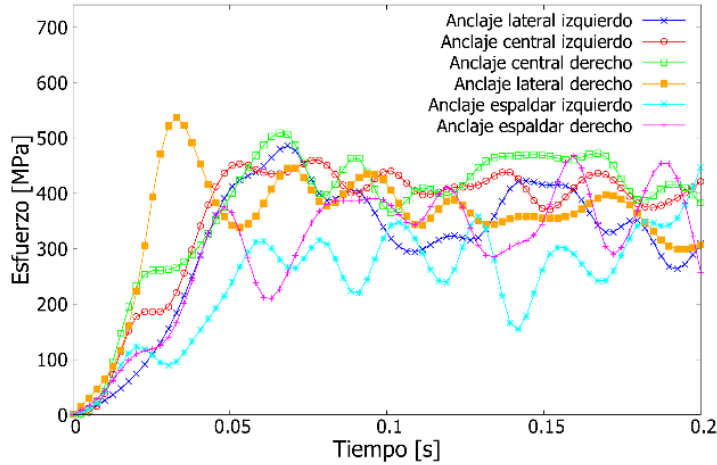

(b)

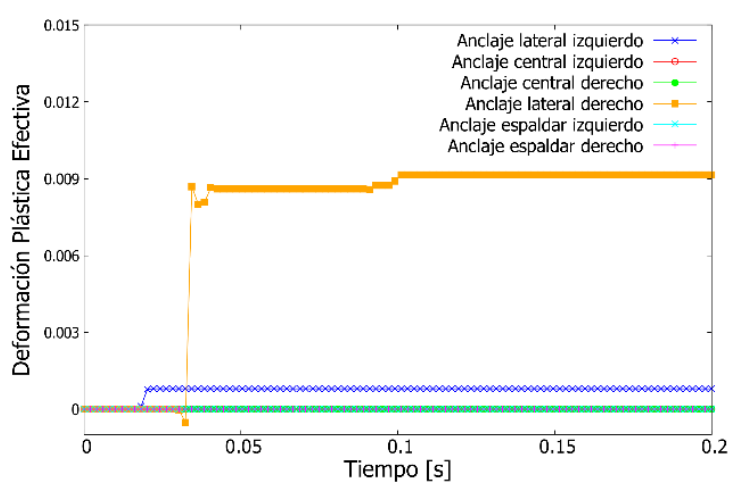

(c)

Figura 7. Comportamiento de los anclajes. (a) Esfuerzos en los anclajes modificados del cinturón de 2 puntos (b) Esfuerzos en los anclajes modificados del cinturón de 3 puntos (c)

Deformación plástica efectiva en los anclajes modificados del cinturón de 3 puntos 
Se mejoró la resistencia del anclaje central a la base colocando independientemente la sujeción del cinturón de la derecha e izquierda; además el cambio de posición del anclaje aumentó el área de conexión entre el anclaje y base disminuyendo la deformación de la estructura base y el desplazamiento del asiento respecto a su posición original. Los anclajes con las modificaciones propuestas resisten el ensayo descrito en la regulación europea ECE-R14 o su equivalente en normativa ecuatoriana NTE INEN 2704.

\section{Conclusiones y recomendaciones}

Se generó un modelo de elementos finitos de un asiento comercial de autobús que bajo las condiciones y simplificaciones realizadas respecto al asiento fabricado, se evaluó bajo la regulación europea ECE R14 para anclajes de cinturones de seguridad de dos y tres puntos de anclaje.

El modelo de asiento que actualmente se fabrica, se analizó mediante dinámica explicita y se verificó que bajo estas condiciones no cumple con la disposición de resistencia mínima de la Regulación ECE R14.

Las modificaciones en el diseño, como la inclusión de placas triangulares de $4 \mathrm{~mm}$ de espesor en las esquinas de la estructura base, permitieron que el asiento cumpla con lo dispuesto en la regulación ECE R14. Una vez realizadas dichas modificaciones, el esfuerzo equivalente máximo alcanzado (290 MPa) se presentó en el anclaje lateral derecho, se presume debido a que la sujeción del asiento a la estructura de la carrocería del autobús en esta zona es más rígida. Se procuró que las modificaciones sean mínimas de tal manera que no afecten al proceso constructivo del asiento, así como a su ensamblaje, instalación y comercialización.

Se generó una metodología para que las industrias que fabrican asientos en el Ecuador puedan realizar modificaciones mínimas de tal manera que sus diseños se ajusten a regulaciones reconocidas globalmente como la ECE R14.

\section{Agradecimientos}

Los autores agradecen a la Dirección de Investigación de la Universidad Técnica de Ambato por haber financiado esta investigación mediante Resolución del Honorable Consejo Universitario 0313-CU-P2016.

\section{Bibliografía}

ANT. (2016). Total Siniestros, Lesionados y Fallecidos. Agencia Nacional de Tránsito, 1, 2016.

Bank, W. (2015). Mortality caused by road traffic injury (per 100,000 people). World Development Indicators, 1, 2015.

Heo, U., Kim, S.-K., Song, M.-J., Yang, I.-Y., \& Im, K.-H. (2011). Effectiveness evaluation for seat parts by seat belt anchorage strength analysis. International Journal of Precision Engineering and Manufacturing, 12(6), 1031-1034.

Huelke, D. F., \& Compton, C. P. (1995). The effects of seat belts on injury severity of front and rear seat occupants in the same frontal crash. Accident Analysis and Prevention, 27(6), 835-838.

INEC. (2016). Compendio Estadístico, 1, 240-248.

ISO6892. (2016). Materiales metálicos. Ensayo de tracción. Parte 1: Método de ensayo a temperatura ambiente. ISO, 1, 2016.

Jung, H. J., Cho, Y. H., Lee, D. S., Oh, J. C., \& Kwon, Y. D. (2012). a Study of Optimum Design and Analysis With D.O.E for Automotive Seat Frame. International Journal of Modern Physics: Conference Series, 6, 594-600. https://doi.org/10.1142 /S2010194512003832 
Li, Y., Peng, H., Rong, B., Men, Y., \& Zhao, F. (2013). Analysis and Improvement for the Failure of Seat Belt Anchor of a Car Based on Simulation and Experiment. In Proceedings of the FISITA 2012 World Automotive Congress (pp. 717-731). Berlin, Heidelberg: Springer Berlin Heidelberg.

NHTSA. (2015). Lives Saved by Vehicle Safety Technologies and Associated Federal Motor Vehicle Safety Standards, 1960 to 2012. U.S. Department of Transportation, DOT HS $812,525$.

Ogundele, O. J., Ifesanya, A. O., Adeyanju, S. A., \& Ogunlade, S. O. (2013). The impact of seat-belts in limiting the severity of injuries in patients presenting to a university hospital in the developing world. Niger Med J, 54(1), 17-21.

Olschinka, C., Schumacher, A., \& Riedel, D. (2006). Dynamic Simulation of Flight Passenger Seats. In 5th LS-DYNA Anwenderforum (pp. 41-58). Retrieved from https://www.dynamore.de/de/download/papers/forum06/passive-safety/dynamicsimulation-of-flight-passengerseats\%5Cnhttps://www.dynamore.de/de/news/pressemitteilungen/pre2011/LSDYNA-Conference-in-Ulm-was-great-success.-This

Patil, P. M., \& Kumar, M. S. (2016). Lightweight Options for Seat Structure in a Bus. Journal of Advanced Engineering Research, 3(1), 72-77.

Reyes, V., Rosales, C., Guzmán, A., \& Báez, S. (2017). Estudio del cumplimiento de la norma INEN 2205 en los asientos de buses de transporte público urbano ( Study of the compliance of the INEN 2205 standard in the seats of urban public transport bus. Enfoque UTE, 8(5), 76-88.

Shi, P., Wang, S., \& Xiao, P. (2017). Strength Analysis on Safety-Belt ISOFIX Anchorage for Vehicles Based on HyperWorks and Ls-Dyna. In D. Liu, S. Xie, Y. Li, D. Zhao, \& E.-S. M. El-Alfy (Eds.), Neural Information Processing (pp. 387-396). Cham: Springer International Publishing.

Siwadamrongpong, S., Rooppakhun, S., Burakorn, P., \& Murachai, N. (2013). Strength Analysis of the Seat Structure for Large Passenger Vehicles by Using Finite Element Method (Vol. 658, pp. 335-339). Trans Tech Publications.

UNECE. (2015). Uniform provisions concerning the approval of vehicles with regard to safety-belt anchorages, ISOFIX anchorages systems, ISOFIX top tether anchorages and i-Size seating positions (2015/1406), 1.

Veeranjaneyulu, M., \& Ravikanth, D. (2015). Impact Strength Analysis on Passenger Seat during Crash by Using Hyper Works. International Journal of Research, 2(10), 10841088.

Yuce, C., Karpat, F., Yavuz, N., \& Sendeniz, G. (2014). A Case Study: Designing for Sustainability and Reliability in an Automotive Seat Structure. Sustainability, 6(7), 4608-4631. 